\title{
Some functorial properties of Schatten classes.
}

\author{
Lav Kumar Singh • A.K. Verma
}

Received: date / Accepted: date

\begin{abstract}
In this paper, we begin with the study of elements in $C^{*}$-algebras which are mapped to Schatten class ideals through the faithful left regular representation. We further give some functorial properties of Schatten classes on the category of representations of a $C^{*}$-algebra and category of unitary representations of a group.
\end{abstract}

Keywords Schatten classes · Representations · Categories · Functors

Mathematics Subject Classification (2010) 47B10 · 46M99

\section{Introduction}

We aim to explore how Schatten classes on Hilbert spaces influence the representations of $C^{*}$-algebras or locally compact Hausdorff groups. We begin our study in the second section with the left regular representation of $L^{\infty}(X)$ on $L^{2}(X)$, where $(X, \mathcal{B}(X), \mu)$ is a $\sigma$-finite measure space. We give a few conditions on measure properties of $X$ such that the pullback of $p^{t h}$-Schatten ideal is not trivial (zero). This establishes the fact that these pullbacks are not trivially zero. Then in the third section, we give a nice functor on category

The research work presented here was jointly carried out by the authors while being funded by Council of Scientific and Industrial Research- Government of India and University Grants Commission-Government of India.

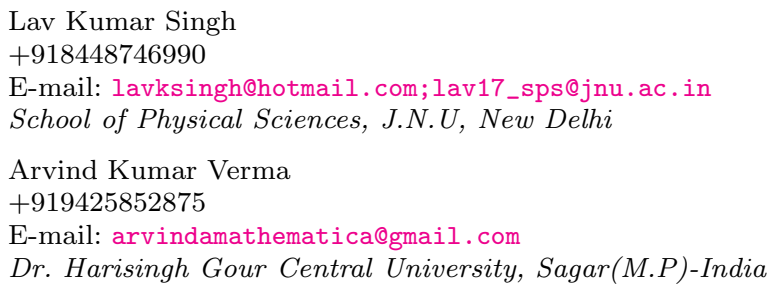


of representations of a $C^{*}$-algebra and category of representations of a group which is associated to Schatten classes. Let us begin with a few preliminaries.

\subsection{Measure space}

Let $(X, \mu)$ be a measure space. A set $A \subset X$ is said to be atom of $\mu$ if $\mu(A)>0$ and for every $B \subset A$, either $\mu(B)=0$ or $\mu(B)=\mu(A)$. Subsets of $X$ which are not atoms are said to be diffuse. A measure is said to be atomic if there exists atleast one atom, else the measure is said to be without atom.

\subsection{Schatten classes on Hilbert spaces}

Let $\mathcal{H}$ be Hilbert space and $T \in B(\mathcal{H})$. For $p \in[1, \infty)$ define Schatten p-norm of $T$ as

$$
\|T\|_{p}=\sum_{n \geq 1}\left(s_{n}^{p}(T)\right)^{1 / p}
$$

where $s_{1}(T) \geq s_{2}(T) \geq \ldots s_{n}(T) \geq 0$ are the singular values of operator $|T|$. Clearly $\|T\|_{p}=\operatorname{tr}\left(|T|^{p}\right)$. The p-th Schatten class operators are those $T \in B(\mathcal{H})$ for which $\|T\|_{p}<\infty$. Let $S_{p}(\mathcal{H})$ denotes the collection of all pth Schatten class operators in $B(\mathcal{H})$. Then $S_{p}(\mathcal{H})$ is an ideal of $B(\mathcal{H})$ and $\|\cdot\|_{p}$ is a norm on $S_{p}(\mathcal{H})$ which makes it a Banach *-algebra (involutions are adjoint maps). Further, we have the containment $S_{p}(\mathcal{H}) \subset S_{q}(\mathcal{H})$ for each $q>p$. The Schatten p-norm satisfies $\|\cdot\|_{q} \leq\|\cdot\|_{p}$ for all $q \geq p$. Operators in $S_{p}(\mathcal{H})$ are compact, and $S_{p}(\mathcal{H})$ contains all finite rank operators. $S_{p}(\mathcal{H})$ is reflexive for $1<p<\infty$ since they are uniformly convex. We also know that $S_{p}(\mathcal{H})^{*}=S_{q}(\mathcal{H})$, where $\frac{1}{p}+\frac{1}{q}=1$. The ideal $S_{2}(\mathcal{H})$ is a Hilbert space with inner product $\langle A, B\rangle=\operatorname{Tr}\left(B^{*} A\right)$. A detailed discussion about these facts can be found in [1]. We will use the notation $S_{\infty}(\mathcal{H})=B_{0}(\mathcal{H})$ for the algebra of compact operators.

1.3 Representations of $C^{*}$ algebras and locally compact groups.

A representation of a $C^{*}$-algebra $\mathcal{A}$ is a continuous $*$-homomorphism $\mathcal{A} \rightarrow$ $B(\mathcal{H})$ for some Hilbert space $\mathcal{H}$. Injective representations are called faithful representations. Gelfand Naimark theorem states that every $C^{*}$-algebra possess a faithful representation on some Hilbert space.[6, Ch.3]

A unitary representation of a locally compact Hausdorff group $G$ is a homomorphism $G \rightarrow U(\mathcal{H})$ for some Hilbert space $H$, continuous with respect to strong topology on $U(\mathcal{H})$, where $U(\mathcal{H})$ denotes the group of unitary operators on $\mathcal{H}[5$, Ch.3]. 


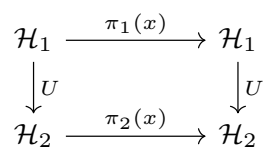

Fig. 1

\subsection{Categories}

By $\mathbf{B a n}_{1}$, we denote the category whose objects are Banach spaces and morphisms are contractive linear maps. $\mathcal{R} \operatorname{ep}(\mathcal{A})$ denotes the category whose objects are representations of $C^{*}$-algebra $\mathcal{A}$ and natural transformations generated by intertwining operators define morphisms, i.e. $\phi: \pi_{1} \rightarrow \pi_{2}$ is a morphism between two representations $\pi_{1}: \mathcal{A} \rightarrow B\left(\mathcal{H}_{1}\right)$ and $\pi_{2}: \mathcal{A} \rightarrow B\left(\mathcal{H}_{2}\right)$, then there exists an operator $U: \mathcal{H}_{1} \rightarrow \mathcal{H}_{2}$ such that for each $x \in \mathcal{A}$ the diagram in fig. 1 commutes.

Similarly, we denote the category of unitary representations of a group $G$ by $\mathcal{R} e p(G)$ where natural transformations generated by intertwining operators define morphisms.

An assignment $\mathcal{F}: \mathcal{C} \rightarrow \mathcal{D}$, where $\mathcal{C}, \mathcal{D}$ are any two categories, is said to be a functor if it assigns to each object $C \in \mathcal{C}$ a unique object $\mathcal{F}(C) \in \mathcal{D}$ and to each morphism $f: C_{1} \rightarrow C_{2}$ in $\mathcal{C}$ a unique morphism $\mathcal{F}(f): \mathcal{F}\left(C_{1}\right) \rightarrow \mathcal{F}\left(C_{2}\right)$ in $\mathcal{D}$ such that $\mathcal{F}\left(\operatorname{Id}_{\mathrm{C}}\right)=\operatorname{Id}_{\mathcal{F}(C)}$ and $\mathcal{F}(g \circ f)=\mathcal{F}(g) \circ \mathcal{F}(f)$ for any pair of morphisms $f, g$ in $\mathcal{C}$ (given that they can be composed).

\section{Schatten type functions in $L^{\infty}(X)$.}

Let $X$ be a measure space with $\sigma$-finite Borel measure $\mu$. Consider the faithful representation of $C^{*}$-algebra $L^{\infty}(X)$ by

$$
\pi: L^{\infty}(X) \rightarrow B\left(L^{2}(X)\right)
$$

such that $\pi(f) g=f . g$ for each $f \in L^{\infty}(X)$ and $g \in L^{2}(X)$. For each $1 \leq p \leq$ $\infty$, we define the set

$$
S_{p}(X)=\pi^{-1}\left(S_{p}\left(L^{2}(X)\right)\right),
$$

which is a collection of all the functions in $L^{\infty}(X)$ which are mapped to Schatten $p$-class operators on Hilbert space $L^{2}(X)$. Clearly, $S_{p}(X)$ is an ideal in $L^{\infty}(X)$.

Lemma 1 For group $\mathbb{Z}$ with Haar measure(counting measure), $S_{p}(\mathbb{Z})=\ell^{p}(\mathbb{Z})$ for all $1 \leq p \leq \infty$.

Proof Consider the standard orthonormal basis $\left\{e_{i}\right\}_{i \in \mathbb{Z}}$ of $\ell^{2}(\mathbb{Z})$. Let $f \in \ell^{\infty}$. Then $f \in S_{p}(\mathbb{Z})$ if and only if

$$
\|\pi(f)\|_{p}=\sum_{n \in \mathbb{Z}}\left\langle|f(n)|^{p} e_{n}, e_{n}\right\rangle=\sum_{n \in \mathbb{Z}}|f(n)|^{p}<\infty
$$


Hence $S_{p}(\mathbb{Z})=\ell^{p}(\mathbb{Z})$ for all $1 \leq p<\infty$. Case $p=\infty$ is trivial.

Remark 1 The collection $S_{p}(X)$ is not independent of the faithful representation. For example, consider the faithful representation $\pi \otimes \mathbb{1}: \ell^{2}(\mathbb{Z}) \rightarrow$ $B\left(\ell^{2}(\mathbb{Z}) \otimes \ell^{2}(\mathbb{Z})\right)$. In this case, none of the sequences is mapped to trace class operator.

Lemma 2 For group $\mathbb{R}$ with Haar measure, $S_{p}(\mathbb{R})=\{0\}$ for all $1 \leq p<\infty$ and $S_{\infty}(\mathbb{R})=L^{\infty}(\mathbb{R})$. Even for compact group $\mathbb{S}^{1}$ with Haar measure, $\bar{S}_{p}\left(\mathbb{S}^{1}\right)=$ $\{0\}$ for $1 \leq p<\infty$ and $S_{\infty}\left(\mathbb{S}^{1}\right)=L^{\infty}\left(\mathbb{S}^{1}\right)$.

Proof Consider the Gabor orthonormal basis $\left\{e_{n, m}\right\}_{n, m}$ of $L^{2}(\mathbb{R})$, where $e_{n, m}(x)=e^{2 \pi i m x} \chi_{[n, n+1]}$. Let $f \in L^{\infty}(\mathbb{R})$ and $1 \leq p<\infty$. Then $f \in S_{p}(\mathbb{R})$ if and only if

$$
\|\pi(f)\|_{p}=\sum_{n, m \in \mathbb{Z}} \int_{\mathbb{R}}|f|^{p} e_{n, m} \cdot \overline{e_{n, m}} d \mu=\sum_{n, m} \int_{n}^{n+1}|f|^{p} d \mu<\infty .
$$

This is unconditional sum of positive numbers which converges if and only if $\int_{\mathbb{R}}|f|^{p} d \mu=0$ and hence if and only if $f=0$.

Similarly, using the orthonormal basis $\left\{e^{2 \pi i n x}\right\}_{n \in \mathbb{Z}}$ for $L^{2}\left(\mathbb{S}^{1}\right)$, one can prove that $f \in S_{p}\left(\mathbb{S}^{1}\right)$ if and only if $f=0$. The $p=\infty$ situation is easily seen to be true in both cases.

Remark 2 The above examples may lead us to the hypothesis that $S_{p}(X)$ is non-trivial if and only if $X$ is discrete, which is not true as proved below.

Theorem 1 For a measure space $X$ with a $\sigma$-finite Borel measure $\mu$, the ideal $S_{p}(X)$ for $1 \leq p<\infty$ is trivial(zero) if and only $\mu$ is without atom. ${ }^{1}$

Proof Suppose $(X, \mu)$ is atomic. Then we have $X=F \cup\left(\cup_{x \in D}\{x\}\right)$, where $F$ is the diffuse set(without atoms) and $D$ is the set of all atoms (atoms in regular Borel measure are always almost singleton and countable[2, 2.2]). Let $f=\sum_{x \in D} \beta_{x} \chi_{x}$ such that $\sum_{x \in D}\left|\beta_{x}\right|^{p}<\infty$. Notice that the set $\left\{\frac{1}{\mu(x)^{1 / 2}} \chi_{x}\right\}$ is orthonormal. Hence,

$$
\|\pi(f)\|_{p}=\sum_{x \in D}\left|\beta_{x}\right|^{p}<\infty
$$

It follows that $f \in S_{p}(X)$ and

$$
S_{p}(X)=\left\{f \in L^{\infty}(X): \operatorname{supp}(\mathrm{f}) \subset D \text { and } \sum_{x \in D}|f(x)|^{p}<\infty\right\}
$$

for all $1 \leq p<\infty$. Conversely, $S_{p}(X)$ is non-empty for all $1 \leq p<\infty$. Let $f \in S_{p}(X)$ for a fixed $p$. Then $\pi(f)$ is compact(all Schatten class operators are compact) and $\sigma(\pi(f))=\overline{f(X)}$ must be finite or a set with limit point 0 .

\footnotetext{
1 This proof is motivated by the idea provided by Prof. Martin Argerami.
} 
So $f$ must be a simple function, $f=\sum_{j} \alpha_{j} \chi_{E_{j}}$. Here projection $\pi\left(\chi_{E_{j}}\right)$ must be finite rank except when $\alpha_{j}=0$. Now, if the set $D$ of atoms is empty then $\mu\left(E_{j} \cap F\right)>0$ for every single $j$ and this will force that $\chi_{E_{j}}$ is not finite rank. Thus, $D$ must be non-empty.

Remark 3 Notice that $S_{p}(\mathbb{R})$ and $S_{p}\left(\mathbb{S}^{1}\right)$ were empty for $1 \leq p<\infty$ because the Haar measure on $\mathbb{R}$ and $\mathbb{S}^{1}$ are non-atomic. $S_{p}(\mathbb{Z})$ was non-empty because $\mathbb{Z}$ with counting measure is atomic (singletons are atoms). There are in fact compact non-discrete groups which are atomic. For example, consider the infinite product $\Pi_{J} G$ with counting measure, where $G$ is a finite group with discrete topology. Clearly, $\Pi_{J} G$ is a non-discrete compact group which is atomic (singletons are the atoms, and diffuse is empty). For groups with left/right invariant measures, even stronger result holds.

Theorem 2 Let $G$ be a group equipped with left/right invariant $\sigma$-finite Borel measure $\mu$. Then $S_{p}(G)$ for $1 \leq p<\infty$ is non-empty if and only if $\mu$ is counting measure (up to product with scalars) and $G$ is a countable group.

Proof From Theorem 1. we know that $S_{p}(G)$ is non-empty if and only if $\mu$ is atomic. Let $\{x\}$ be any atom. Then $\mu(x)>0$. By left/right translation invariance we know that $\mu(\{g\})=\mu(\{x\})$ for all $g \in G$. Hence each singleton in $G$ is an atom. However, atoms in a $\sigma$-finite measure space are countable [2, 2.2,ex.11]. Thus $G$ must be a countable group in which all singletons are atoms of the same measure. Hence $\mu$ must be some scalar multiple of counting measure.

\section{Functors associated to Schatten classes}

\subsection{Category of representations of a $C^{*}$-algebra.}

The above constructions characterize the family of spaces(with measure) for which $S_{p}(X)$ is non-empty. Studying $S_{p}(X)$ for these spaces results in some abstract non-sense. We take a short exact sequence of Banach spaces, as defined in [3]. Consider the category of Banach spaces, $\mathbf{B a n}_{1}$. For each $1 \leq p<\infty$, we get an exact sequence $E_{p}$ -

$$
0 \longrightarrow S_{p}(X) \stackrel{\pi}{\longrightarrow} S_{p}\left(L^{2}(X)\right) \longrightarrow \frac{S_{p}\left(L^{2}(X)\right)}{\pi\left(S_{p}(X)\right)} \longrightarrow 0
$$

Also for each pair $(p, q)$ such that $1 \leq p<q<\infty$, we have the natural contractive injections -

$$
i_{p, q}: S_{p}\left(L^{2}(X)\right) \rightarrow S_{q}\left(L^{2}(X)\right) \quad \text { and } \quad \pi^{-1} \circ i_{p, q} \circ \pi: S_{p}(X) \rightarrow S_{q}(X)
$$

It is easy to verify that $\left\{E_{p}\right\}_{p \geq 1}$ forms a directed system in the category of exact sequences of Banach spaces (morphism between two exact sequences $E_{p}$ and $E_{q}$ for $q>p$, denoted by $\phi_{p, q}$, are required to be contractions between the corresponding nodes) 


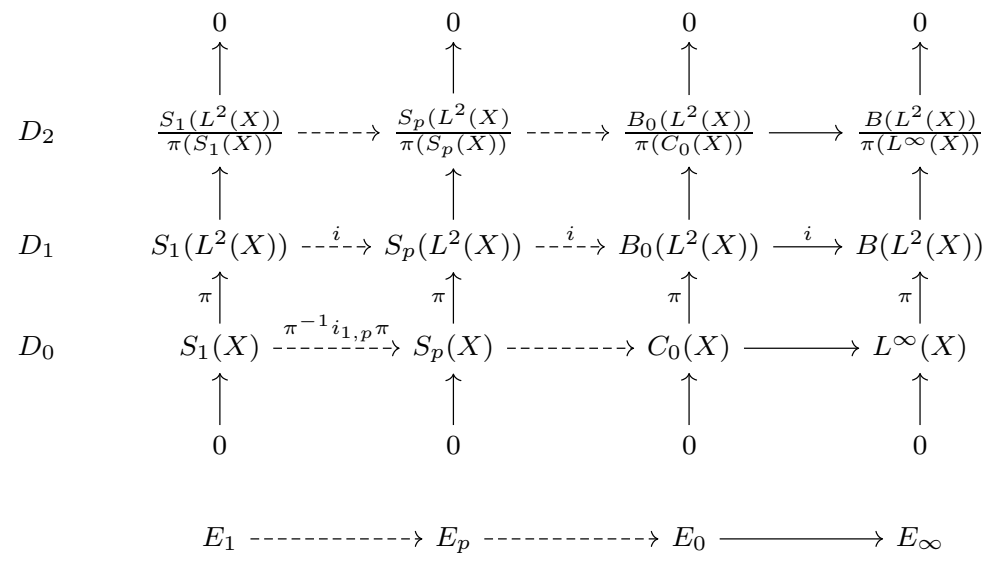

Fig. 2

Lemma 3 The pair $\left\{E_{p}, \phi_{p, q}\right\}$ forms a directed system on the set $[1, \infty)$ in the category of exact sequences of Banach spaces.

Proof This is just an easy verification exercise. Fig. 2 gives us a complete picture. Vertical columns are short exact sequences and each row, $D_{0}, D_{1}$ and $D_{2}$ forms a directed system in the category of Banach spaces. Notice that each rectangular block commutes.

As we mentioned in remark 1 , the definition of $S_{p}(X)$ depends on the left regular representation of $L^{\infty}(X)$. Therefore, it leads us to study the more general case. Let $\mathcal{A}$ be a $C^{*}$-algebra, and $\pi: \mathcal{A} \rightarrow B\left(\mathcal{H}_{\pi}\right)$ be a representation(not necessarily faithful) of $\mathcal{A}$. We define

$$
S_{p}^{\pi}(\mathcal{A})=\pi^{-1}\left(S_{p}\left(\mathcal{H}_{\pi}\right)\right) .
$$

Now, we have an exact sequence $E_{p}^{\pi}$, and for $p>q$ we have the following morphism between $E_{p}^{\pi} \rightarrow E_{q}^{\pi}$ arising through natural maps.

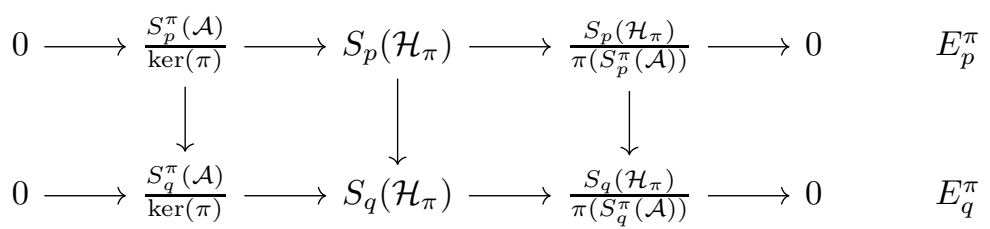

Theorem 3 Schatten classes on Hilbert spaces gives a functor from the category $\mathcal{R e p}(\mathcal{A})$ of faithful representations of $C^{*}$-algebra $\mathcal{A}$ to the category $D S_{[1, \infty)}$ of directed systems on the directed set $\{p: 1 \leq p<\infty\}$.

Proof The assignment $\mathcal{S}: \mathcal{R} \operatorname{ep}(\mathcal{A}) \rightarrow D S_{[1, \infty)}$ given by

$$
S(\pi)=E^{\pi}
$$


is a well-defined functor, where $E^{\pi}$ is the directed system $\left\{E_{p}^{\pi}\right\}_{p \geq 1}$

$$
E_{1}^{\pi} \rightarrow \ldots E_{p}^{\pi} \rightarrow \ldots
$$

\subsection{Category of group representations.}

Let $G$ be a locally compact Hausdorff group. Consider the unitary representation $\pi: G \rightarrow U\left(\mathcal{H}_{\pi}\right)$ of $G$ on a Hilbert space $\mathcal{H}_{\pi}$. Consider the induced non-degenerate representation $\pi: L^{1}(G) \rightarrow B\left(\mathcal{H}_{\pi}\right)$ of Banach-* algebra $L^{1}(G)$ $[5,3.2]$, which is given by

$$
\langle\pi(f) u, v\rangle=\int_{G} f(x)\langle\pi(x) u, v\rangle d x .
$$

Now, we define

$$
T_{p}^{\pi}(G)=\pi^{-1}\left(S_{p}\left(\mathcal{H}_{\pi}\right)\right)
$$

the pullback (through $\pi$ ) of Schatten p-class operators on $\mathcal{H}_{\pi}$. Clearly, $T_{p}^{\pi}(G)$ is an ideal in $L^{1}(G)$.

With this notion, we get a functor associated Schatten classes as described in next theorem.

Theorem 4 Let $G$ be a locally compact Hausdorff group. Associated to Schatten classes, there is a functor $\mathcal{S}: \mathcal{R e p}(G) \rightarrow D S_{[1, \infty)}$.

Proof This is again an easy verification that $\pi \rightarrow\left\{E_{p}^{\pi}\right\}_{p \geq 1}$ is a functor. Where $E_{p}^{\pi}$ is the exact sequence given by

$$
0 \longrightarrow \frac{T_{p}^{\pi}(G)}{\operatorname{ker}(\pi)} \longrightarrow S_{p}\left(\mathcal{H}_{\pi}\right) \longrightarrow \frac{S_{p}\left(\mathcal{H}_{\pi}\right)}{\pi\left(T_{p}^{\pi}(G)\right)} \longrightarrow 0
$$

and the morphisms are again the natural ones similar to the ones considered in Theorem 3.

\subsection{Some problems}

We end this note with a few problems.

1. Can we give a condition on topology of $G$, similar to the condition on measure on $X$ in Theorem 1, for the left regular representation $\pi$ of a locally compact Hausdorff group $G$ such that the $\pi^{-1}\left(S_{p}\left(L^{2}(G)\right)\right)$ is nonempty?

2. It is shown in [4, Th. 4.1] that direct limits of a directed system of Banach spaces exist in the category $\operatorname{Ban}_{1}$. Its also been shown in the remark after [7, Def. 2.4] that the filtered limits may not be exact. Does filtered limit from Fig. 2

$$
0 \rightarrow \underset{D_{0}}{\longrightarrow} \rightarrow \stackrel{D_{1}}{\longrightarrow} \rightarrow \stackrel{D_{2}}{\longrightarrow} \rightarrow 0
$$

preserve the exactness? If yes then what about the same problem with arbitrary representation $\pi$ ? 
Acknowledgements We would like to acknowledge the financial support provided by C.S.I.R and U.G.C in form of Junior Research Fellowship.

\section{References}

1. Palmer, Theodore W. Banach algebras and the general theory of $*$-algebras. Cambridge University Press, Vol. 1,2 (2001).

2. Chung, Kai Lai, A Course in Probability Theory, Academic Press, 1974.

3. Castillo, J., \& Moreno, Y. (2006). The category of exact sequences of Banach spaces. In J. Castillo \& W. Johnson (Eds.), Methods in Banach Space Theory (London Mathematical Society Lecture Note Series, pp. 139-158). Cambridge: Cambridge University Press.

4. Jesus M Castillo, The Hitchhiker Guide to Categorical Banach Space Theory, Extracta Mathematicae Vol. 25, No. 2, 103 - 149 (2010).

5. Folland, Gerald B., A course in abstract harmonic analysis, Taylor \& Francis group, 2016 .

6. Murphy, Jerald J., C*-Algebras and Operator Theory, Academic Press, 2014.

7. Kobi Kremnizer, Craig Smith, A Tannakian Reconstruction Theorem for IndBanach Spaces arXiv:1703.05679 [math.FA]. 